\title{
Drosophila melanogaster Lacking the Lysosomal Enzyme Palmitoyl-Protein Thioesterase 1 Accumulate Aberrant Ultrastructural Deposits in the Form of Laminar Cytoplasmic Bodies
}

\author{
A. J. Hickey*, J. G. Ault**, C. A. Korey***, M. E. MacDonald****, and R. L. Glaser**** \\ *Department of Biomedical Sciences, University at Albany, State University of New York, Center \\ for Medical Science, 150 New Scotland Avenue, Albany, NY 12208 \\ **Wadsworth Center, New York State Department of Health, P.O. Box 22002, Albany, NY 12201- \\ 2002 \\ *** Department of Biology, College of Charleston, 66 George Street, Charleston, SC 29424 \\ ****Molecular Neurogenetics Unit, Massachusetts General Hospital, Bldg 149, 13th Street, \\ Charlestown, MA 02129
}

The gene PPT1 encodes the lysosomal enzyme palmitoyl-protein thioesterase 1 (Ppt1), which cleaves long-chain fatty acids from acylated proteins. Mutations in the human PPT1 gene cause the pediatric neurodegenerative disease infantile neuronal ceroid lipofuscinosis (INCL) [1]. INCL is characterized by the accumulation of granular osmiophilic deposits (GROD) in all cells and the extensive death of cortical neurons [2]. It is not known how the loss of Ppt1 causes neurodegeneration or GROD formation. Drosophila melanogaster has been a useful model system for studying numerous genetic neurodegenerative diseases, and Drosophila has a functional PPTI ortholog (Ppt1), so it was hypothesized that Drosophila could provide a useful model to study the etiology of INCL [3-4].

A chromosomal deletion was generated, $D f(1) 446-20$, which removes Ppt1, in addition to three neighboring genes [4]. Flies carrying the $D f(1) 446-20$ deletion lack Ppt1 enzyme activity, yet are viable, fertile, and have no obvious signs of neurodegeneration. They do, however, accumulate aberrant ultrastructural deposits named laminar cytoplasmic bodies (LCBs). In contrast to the GROD that accumulates in human disease, which is granular and amorphous in structure, LCBs are laminar and spherical in structure and more closely resemble the deposits seen in Tay-Sachs disease [5]. Two major types of LCBs have been observed in $D f(1) 446-20$ fly tissues. One type, found predominantly in females, consist of tightly packed concentric layers of material often surrounding a granular core (Fig. 1). The other type, found predominantly in males, consists of stacked layers of material often separated from one another to form prominant gaps (Fig. 2). LCBs were detected in multiple Drosophila tissues but were enriched in both frequency and size in the adult brain. This contrasts with GROD in humans, which is abundant in all tissues.

The LCB phenotype could be rescued in the brains of transgenic $D f(1) 446-20$ flies by expressing a Ppt 1 cDNA transgene in the central nervous system. In addition, suppressing Ppt1 enzyme activity by expressing a Ppt1 RNAi construct in otherwise wildtype flies resulted in the formation of LCBs. These observations suggest that the LCB phenotype is caused by the loss of Ppt1, specifically, and not by the loss of any of the other three genes removed by the $D f(1) 446-20$ deletion.

Although LCBs do not resemble GROD, the fact that the loss of Ppt1 enzyme activity in both flies and humans results in the abnormal accumulation of material suggests that a common cellular mechanism is disrupted in both organisms. Understanding the mechanism involved in LCB 
formation in flies may provide clues to how and why GROD accumulates in human INCL patients, and may ultimately provide insights into INCL etiology.

References

[1] J. Vesa et al. Nature 376 (1995) 584.

[2] S. L. Hofmann and L. Peltonen, The metabolic and molecular basis of inherited disease, 8th ed. McGraw-Hill, New York, 2001.

[3] M. E. Fortini and N. M. Bonini, Trends Genet. 16 (2000) 161.

[4] R. L. Glaser et al. Gene 312 (2002) 271.

[5] D. P. Perl, Tay-Sachs Disease, Academic Press, San Diego, New York, 2001.

[6] This work was supported by a grant from the National Institute of Neurological Disorders and Stroke to R.L.G.

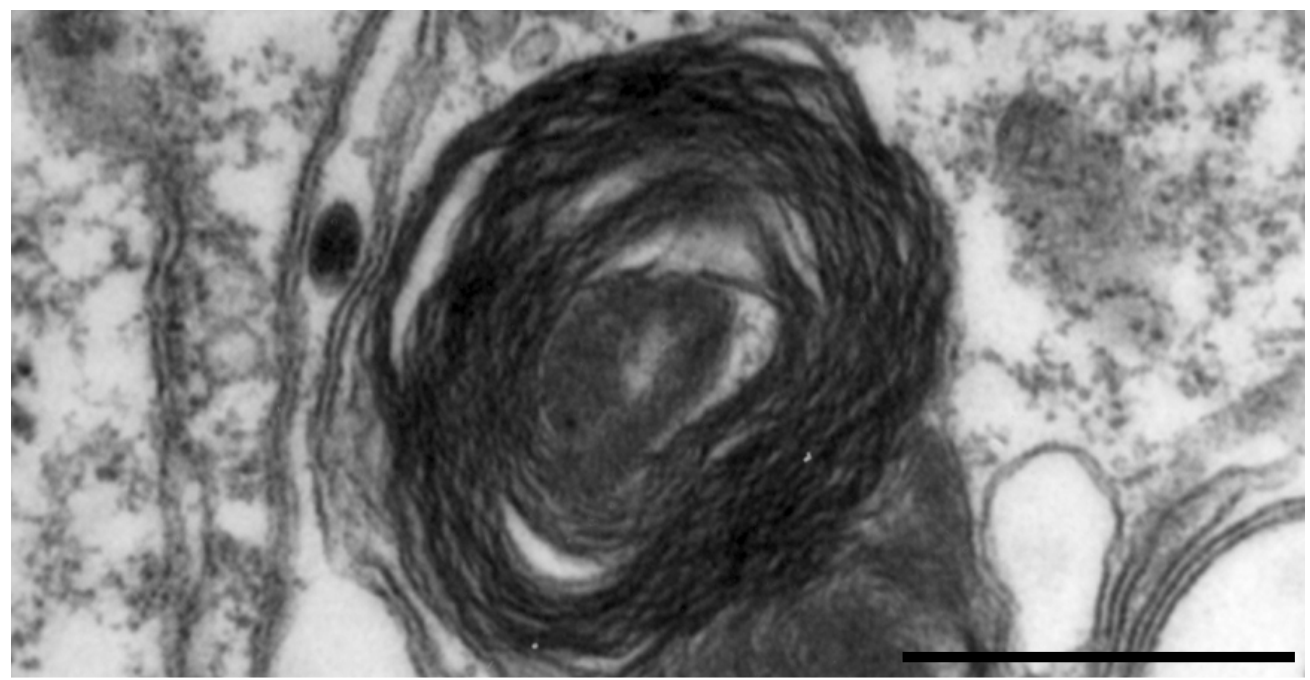

Fig. 1. Laminar cytoplasmic body in the brain of a female $D f(1) 446-20$ fly.

Note concentric layers surrounding a granular core. Scale bar $=1 \mu \mathrm{m}$.

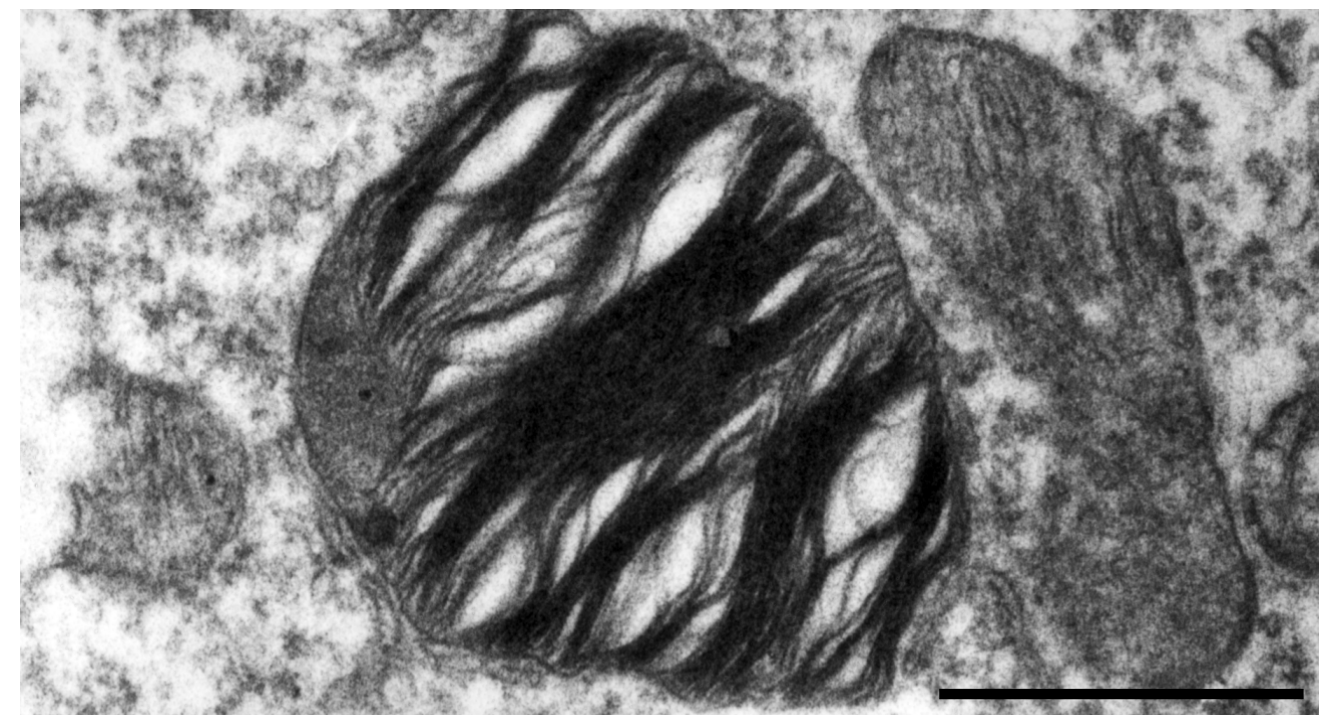

Fig. 2. Laminar cytoplasmic body in the brain of a male $D f(1) 446-20$ fly. Note stacked layers with frequent separations. Scale bar $=1 \mu \mathrm{m}$. 